Polymer Journal, Vol. 2, No. 6, pp 698-708 (1971)

\title{
Stress Relaxation of Blended Polymers of Monodisperse Polystyrenes
}

\author{
Kenkichi Murakami, Katsumichi Ono, Katsuo Shinna,* \\ Taku Ueno, ${ }^{* *}$ and Masato Matsuo*** \\ Chemical Research Institute of Non-aqueous Solutions, \\ Tohoku University, Sendai, Japan.
}

(Received January 27, 1971)

\begin{abstract}
The stress relaxation measurements of some blended polystyrenes were carried out. The blended polymers constructed from monodisperse polystyrenes with different molecular weights were used for this purpose. The effect of the molecular weight distribution on the shape of the relaxation spectrum is discussed. The rubbery regions of the relaxation spectra were characterized by a step-like shape if the molecular weight of each polymer component was larger than the critical molecular weight $M_{\mathrm{c}}$. The molecular weight dependences of various viscoelastic parameters were examined. As for monodisperse polystyrenes, clear relations between the maximum relaxation time $\tau_{\mathrm{m}}$, the steady flow viscosity $\eta_{\mathrm{t}}$, and the molecular weight were observed, while for blended polystyrenes these relations showed some deviations. On the other hand, the viscoelastic parameters may be closely related to one another by the same equations in the cases of both the monodisperse and blended polystyrenes.
\end{abstract}

KEY WORDS Stress Relaxation / Polystyrene / Polymer Blend / Molecular Weight Distribution / Relaxation Spectrum / Maximum Relaxation Time /

As is generally accepted, the relaxation spectrum of an amorphous polymer is represented by two characteristic regions, i.e., "wedge" and "box", regions. The wedge region is associated with the short-time part of the relaxation spectrum, and the molecular theories proposed by $\mathbb{R o u s e},{ }^{7}$ $\mathrm{Zimm}^{2}$ and other workers have been successful in the interpretation of the viscoelastic behaviors of the polymers in this region.

On the other hand, the distribution functions of the relaxation time in the box region are generally characterized by three parameters $\tau_{\mathrm{e}}$, $\tau_{\mathrm{m}}$, and $E_{0}$. The minimum relaxation time $\tau_{\mathrm{e}}$ corresponds to the initial zone of the rubbery region. According to the proposal of Fujita and Ninomiya, ${ }^{3} \tau_{\mathrm{e}}$ is defined as a limiting value of the miximum relaxation time $\tau_{\mathrm{m}}$, when the molecular weight of the polymer converges to $M_{\mathrm{c}}$ (critical molecular weight). As expected from the definition, $\tau_{\mathrm{e}}$ must be independent of molecular weight. Our previous results, ${ }^{4}$ how-

* Takiron Chemical Co., Ltd., Ibo, Hyogo, Japan.

** The Japanese Geon Co., Ltd., Kawasaki, Japan.

*** Bell Telephone Laboratories, Murray Hill, $N$. J., U.S.A. ever, suggest that $\tau_{\mathrm{e}}$ depends upon the molecular weight to some extent.

The maximum relaxation time $\tau_{\mathrm{m}}$ corresponds to the transitional zone between the rubbery region and the liquid flow region. It was shown by one of us ${ }^{5}$ that $\tau_{\mathrm{m}}$ relates to the molecular. weight of a polymer by the following equation,

$$
\log \tau_{\mathrm{m}}=\log A_{\mathrm{g}}-17.44 \frac{T-T_{\mathrm{g}}}{51.6+T-T_{\mathrm{g}}}+3.4 \log n_{w}
$$

or

$$
\log \tau_{\mathrm{m}}=\log A_{\mathrm{s}}-8.86 \frac{T-T_{\mathrm{s}}}{101.6+T-T_{\mathrm{s}}}+3.4 \log n_{w}
$$

Where, $T_{\mathrm{g}}$ is the glass transition temperature, $T_{\mathrm{s}}$ is the characteristic temperature of the Williams-Landel-Ferry equation and $n_{w}$ is the weight-average number of chain links. The value of $\log A$ is a constant depending upon the kind of polymer.

The parameter $E_{0}$ is the height of the box distribution function. It was previously reported that the relative value of $E_{0}$ becomes larger and the shape of the rubbery region of the relaxation spectrum converges to an ideal "box" with increasing sharpness of the molecular weight 
distribution. ${ }^{6,7}$ From our recent results, it becomes clear according to the simple mathematical derivation, that these two criteria have the same origin. $^{8}$

It was thus concluded that the shape of the box part of the relaxation spectrum is closely connected with the molecular weight and the molecular weight distribution of the polymer. In order to investigate these relations in detail, we used monodisperse polystyrenes(PSt) as model polymers which were expected to have the narrow molecular weight distributions. The changes of the shape of the relaxation spectrum, especially those of the rubbery region, are discussed with regard to the composition of blended polymers constructed from these living polymers with different molecular weights.

\section{EXPERIMENTAL}

Monodisperse polystyrenes used in this work were polymerized by $n$-butyllithium catalyst. As would be expected, the values of $\bar{M}_{w} / \bar{M}_{n}$ of these polymers were less than 1.2. Four monodisperse PSt with different molecular weights were prepared as shown in Table I. The viscosityaverage molecular weight $\bar{M}_{v}$ was calculated from the intrinsic viscosity relation as represented by eq 1 (in methyl ethyl ketone at $25^{\circ} \mathrm{C}$ ). ${ }^{9}$

$$
[\eta]=3.9 \times 10^{-4} \bar{M}_{v}^{0.58}
$$

Asterisk shows the molecular weights obtained from sedimentation velocity measurements. Other values of molecular weights were determined by osmometry or light scattering.

Blended polystyrenes with compositions shown in Table II, were prepared from MD-4 polymer (taken as a standard component which will be called $\mathrm{M}_{2}$ hereafter) and MD-1, MD-2 or MD-3 polymer (these three polymers will be called $\mathrm{M}_{1}$ ). All the polymers were obtained by freeze-drying of the benzene solutions of homopolymer mixtures.

The average molecular weights of these blended polymers were calculated from the eq 2 and 3 using the relation between the weight fraction and the molecular weight of the mixture. The molecular weight distributions of the polymer blends listed in Table II are simply the ratio of weight-average value to the number-average one. Therefore, it must be noted that these values show only the apparent molecular weight distributions.

$$
\begin{gathered}
\bar{M}_{w, \mathrm{~b} 1}=\left(w_{1} \bar{M}_{w, 1}+w_{2} \bar{M}_{w, 2}\right) /\left(w_{1}+w_{2}\right) \\
\bar{M}_{n, \mathrm{~b} 1}=\left(w_{1}+w_{2}\right) \bar{M}_{n, 1} \bar{M}_{n, 2} /\left(w_{1} \bar{M}_{n, 1}+w_{2} \bar{M}_{n, 2}\right)
\end{gathered}
$$

where $w$ is the weight fraction of the mixture. Subscripts 1,2 , and b1 were used to denote $M_{1}$,

Table I. Molecular weights and molecular weight distributions of monodisperse polystyrenes

\begin{tabular}{ccccccc} 
Symbol & $M_{n} \times 10^{-4} \mathrm{a}$ & {$[\eta]$} & $\bar{M}_{v} \times 10^{-4}$ & $\bar{M}_{w} \times 10^{-4}$ & $\bar{M}_{n} \times 10^{-4}$ & $\bar{M}_{w} / \bar{M}_{n}$ \\
\hline MD-1 & 1.18 & 0.084 & 1.05 & $1.07^{*}$ & $1.05^{*}$ & 1.02 \\
MD-2 & 5.90 & 0.247 & 6.75 & $7.46^{*}$ & $6.36^{*}$ & 1.17 \\
MD-3 & 11.7 & 0.388 & 14.7 & 18.7 & 18.3 & 1.02 \\
MD-4 & 51.4 & 0.646 & 48.7 & 58.3 & 54.3 & 1.07
\end{tabular}

a Calculated from catalyst concentration.

Table II. Blended polymers prepared from monodisperse polystyrenes

\begin{tabular}{lcccccc}
\hline Symbol & $\mathrm{M}_{1}$ & $\mathrm{M}_{2}$ & $\mathrm{M}_{2} / \mathrm{M}_{1}$ & $\bar{M}_{w} \times 10^{-5}$ & $\bar{M}_{n} \times 10^{-4}$ & Apparent, $\bar{M}_{w} / \bar{M}_{n}$ \\
\hline B-1-1 & MD-1 & MD-4 & $90 / 10$ & 52.6 & 8.73 & 6.02 \\
B-1-2 & MD-1 & MD-4 & $70 / 30$ & 41.1 & 4.51 & 9.11 \\
B-1-3 & MD-1 & MD-4 & $50 / 50$ & 29.7 & 2.10 & 14.15 \\
B-2-1 & MD-2 & MD-4 & $75 / 25$ & 45.7 & 19.80 & 2.30 \\
B-2-2 & MD-2 & MD-4 & $50 / 50$ & 32.9 & 13.20 & 2.49 \\
B-2-3 & MD-2 & MD-4 & $25 / 75$ & 24.3 & 8.25 & 2.95 \\
B-3-2 & MD-3 & MD-4 & $50 / 50$ & 38.5 & 28.40 & 1.36 \\
B-3-3 & MD-3 & MD-4 & $25 / 75$ & 28.6 & 22.50 & 1.27 \\
\hline
\end{tabular}


$\mathbf{M}_{2}$, and blended polymers respectively. After freeze-drying, the sheets of test specimens were prepared by hot-press, and then annealed sufficiently. Since the critical molecular weight $M_{\mathrm{c}}$ of polystyrene was reported as $3.8 \times 10^{4},{ }^{10}$ the relation $\mathrm{M}_{2}>M_{\mathrm{c}}>\mathrm{M}_{1}$ holds for the blended PSt B-1 shown in Table II. Similarly, for the blended PSt B-2 and B-3 apparently $\mathrm{M}_{2}>\mathrm{M}_{1}>M_{\mathrm{c}}$.

The stress relaxation measurements were carried out by using a Shimazu IM-100 "Autograph". The relaxation modulus curves of each sample were plotted at various temperatures and then shifted to construct a master curve according to the well-known time-temperature superposition principle. From this master curve, a relaxation spectrum was obtained by the second approximate method of Ferry and Williams. ${ }^{11}$

\section{RESULTS AND DISCUSSION}

Since the molecular weight of MD-1 is less than $M_{\mathrm{c}}$, it cannot be moulded. As for the

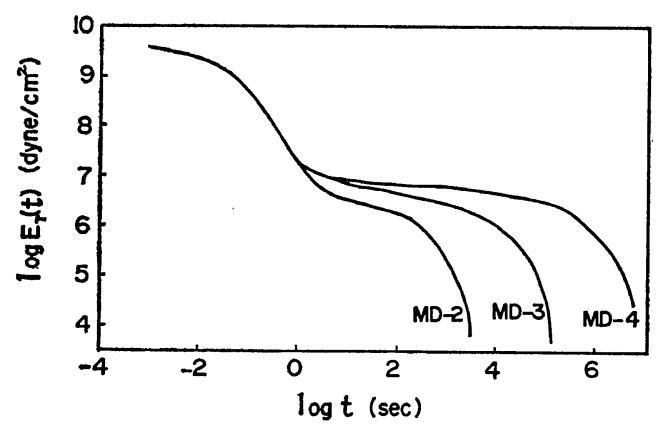

(a)

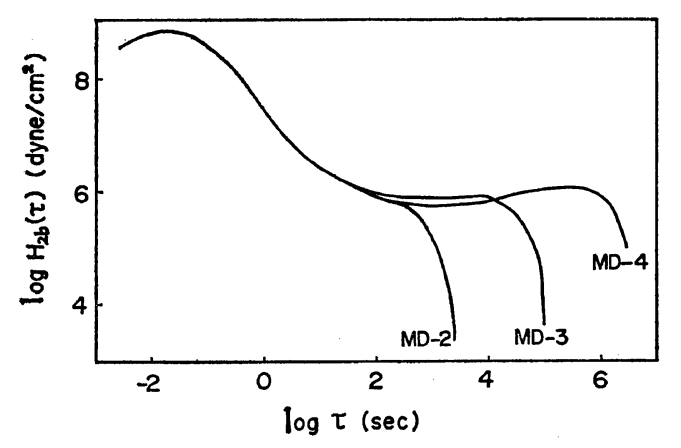

(b)

Figure 1. (a) Stress relaxation master curves for monodisperse polystyrenes reduced to $115^{\circ} \mathrm{C}$. (b) Relaxation spectra for monodisperse polystyrenes.

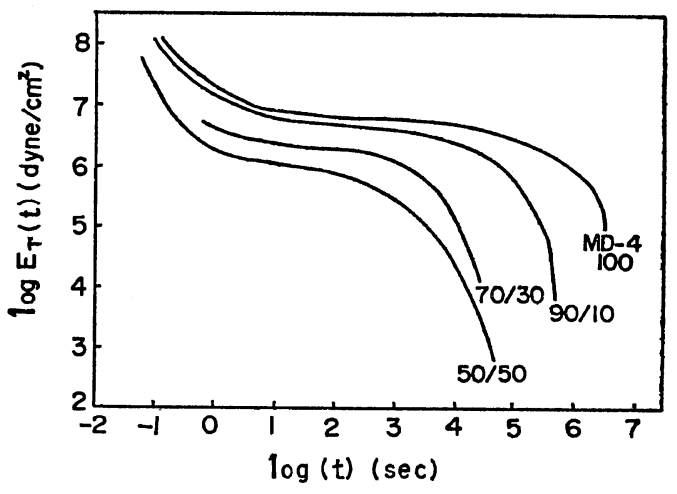

(a)

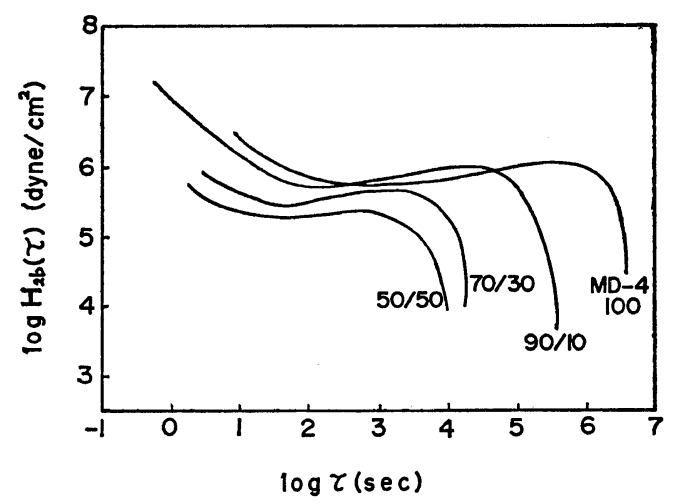

(b)

Figure 2. (a) Stress relaxation master curves for B-1 blends reduced to $115^{\circ} \mathrm{C}$. (b) Relaxation spectra for B-1 blends.

MD-2, MD-3, and MD-4 samples, stress relaxation master curves at a standard temperature $115^{\circ} \mathrm{C}$ were plotted in Figure 1a. Finally relaxation spectra were obtained by the abovedescribed approximation method as shown in Figure 1b.

For the B-1 system, stress relaxation master curves and relaxation spectra were obtained by a similar method with respect to each $M_{1} / M_{2}$ ratio.

Evidently these figures show that all the distribution curves of B-1 polymers are of the simple "box" type. The height of the distribution curve decreases with the decrease of the quantity of $\mathrm{M}_{2}$, and the falling-off portion of the curve tends to shift to the side of the shorter relaxation time. It is noteworthy that the aboveshown changes of the shape of the "box" part 


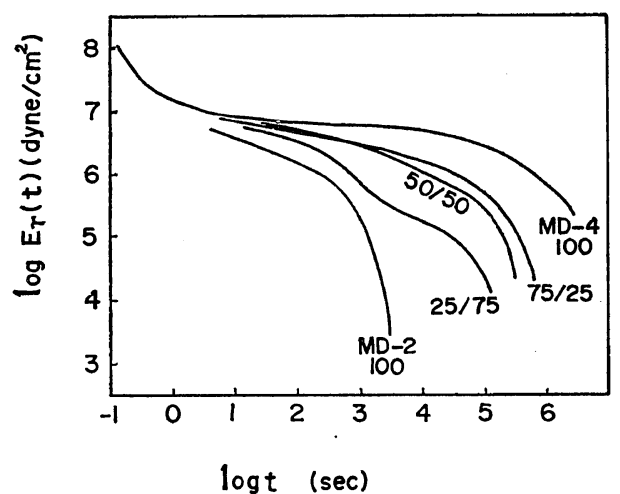

(a)

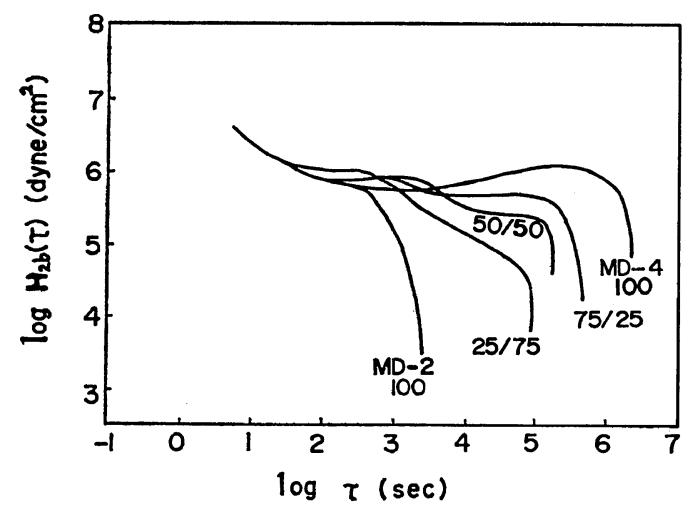

(b)

Figure 3. (a) Stress relaxation master curves for B-2 blends reduced to $115^{\circ} \mathrm{C}$. (b) Relaxation spectra for B-2 blends.

are also observed in the relaxation spectra of homopolymers. ${ }^{6,7}$

For the systems B-2 and B-3 shown in Table II, stress relaxation master curves and relaxation spectra were plotted in Figures 3a, 3b, 4a, and $4 b$.

As shown in Figures 3 and 4, the "wedge" parts of the spectra keep almost the same position, while the "box" parts become step-like in shape.

The shift factors $\log a_{\mathrm{T}}$ for the systems B-1, B-2, and B-3 were plotted against temperature (Figure 5), together with the theoretical curve based on the modified WLF equation as denoted in eq 4 .

$$
\log a_{\mathrm{T}}=-\frac{17.44\left(T-T_{\mathrm{g}}\right)}{51.6+T-T_{\mathrm{g}}}
$$

The good agreement of this WLF curve with the experimental data suggests the fact that even

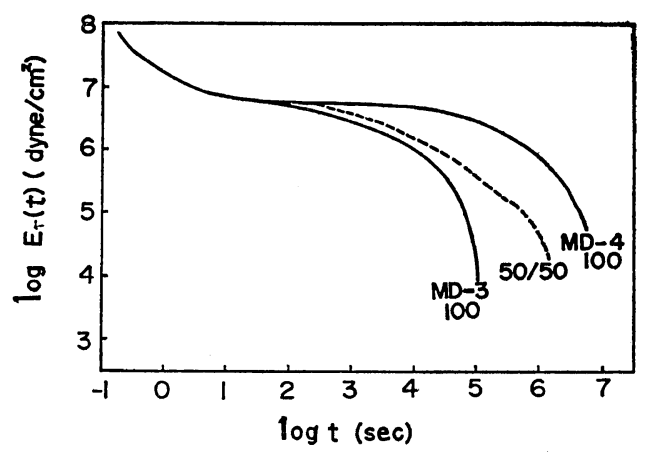

(a)

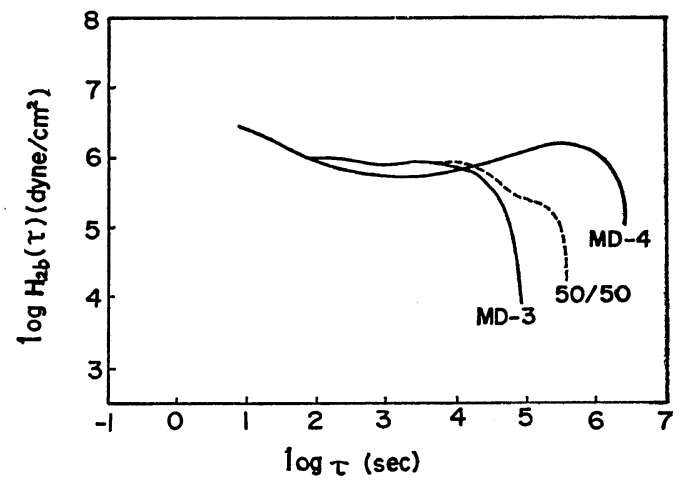

(b)

Figure 4. (a) Stress relaxation master curves for $\mathrm{B}-3$ blends reduced to $115^{\circ} \mathrm{C}$. (b) Relaxation spectra for B-3 blends.

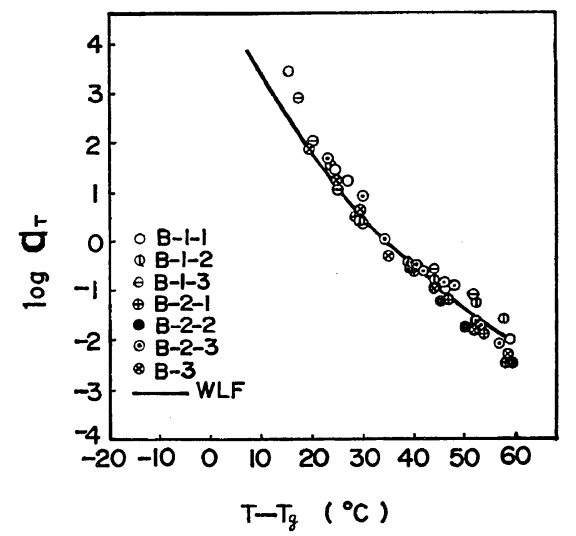

Figure 5. Stress relaxation shift factors for blended polymers of monodisperse polystyrenes. Solid line is the theoretical curve obtained from the WLF equation.

for blended polymers the microstructure is homogeneous, similar to the case of homopolymers.

In the B-1 system, since the molecular weight 
of one component MD-1 of the polymer blend is less than $M_{\mathrm{c}}$, the "box" part may not appear in the relaxation spectrum of this component. Accordingly, the MD-1 component merely acts as a plasticizer to another component MD-4, and the relaxation spectrum of the rubbery region shows the same "box"-type distribution as the homopolymer MD-4. On the other hand, for the systems B-2 and B-3, since the molecular weight of both polymer components is sufficiently larger than $M_{\mathrm{c}}$, each component may show discrete physical properties independently in the viscoelastic behavior of polymer blends.

Let us further discuss the effect of blending on the shape of the relaxation spectra. As shown in Figure 1b, the rubbery regions of the relaxation spectra of monodisperse PSt are well approximated by the box-type distribution function. If we assume an ideal box distribution which is defined by

$$
\begin{array}{ll}
H(\tau)=E_{\mathrm{o}} & \tau_{\mathrm{e}}<\tau<\tau_{\mathrm{m}} \\
H(\tau)=0 & \tau<\tau_{\mathrm{e}}, \quad \tau_{\mathrm{m}}<\tau
\end{array}
$$

the relaxation modulus $E_{\mathrm{r}}(t)$ is calculated from the following equation.

$$
\begin{aligned}
E_{\mathrm{r}}(t) & =\int_{-\infty}^{\infty} H(\tau) \exp (-t / \tau) \mathrm{dln} \tau \\
& =E_{\mathrm{o}}\left[E i\left(-t / \tau_{\mathrm{e}}\right)-E i\left(-t / \tau_{\mathrm{m}}\right)\right]
\end{aligned}
$$

where $E i$ is the exponential integral function.
Table III. Values of $\tau_{\mathrm{m}, 1}$ and $\tau_{\mathrm{m}, 2}$ used for the calculations of the linear mixing of two ideal box distributions

\begin{tabular}{ccc}
\hline No. & $\tau_{\mathrm{m}, 1, \mathrm{sec}}$ & $\tau_{\mathrm{m}, 2}, \mathrm{sec}$ \\
\hline 1 & $10^{2}$ & $10^{3}$ \\
2 & $10^{2}$ & $10^{4}$ \\
3 & $10^{2}$ & $10^{5}$ \\
\hline
\end{tabular}

As the simplest case, suppose the relaxation modulus of a blended polymer is represented by the linear combinations of those of component polymers as follows,

$$
E_{\mathrm{r}, \mathrm{b} 1}(t)=C_{1} E_{\mathrm{r}, 1}(t)+C_{2} E_{\mathrm{r}, 2}(t)
$$

where $C_{1}$ and $C_{2}$ are constants, $E_{\mathrm{r}, b_{1}}(t)$ is the relaxation modulus of the blend and $E_{r, 1}(t)$ and $E_{\mathrm{r}, 2}(t)$ are those of the component polymers. It may be said that the values of $E_{\mathrm{o}}$ and $\tau_{\mathrm{e}}$ do not depend upon the molecular weight in the case of monodisperse polymers. Thus combining eq 6 and 7 ,

$$
\begin{aligned}
E_{\mathrm{r}, \mathrm{b} 1}(t)= & E_{\mathrm{o}}\left(C_{1}+C_{2}\right) E i\left(-t / \tau_{\mathrm{e}}\right) \\
& -E_{\mathrm{o}}\left[C_{1} E i\left(t / \tau_{\mathrm{m}, 1}\right)+C_{2} E i\left(t / \tau_{\mathrm{m}, 2}\right)\right]
\end{aligned}
$$

where $\tau_{\mathrm{m}, 1}$ and $\tau_{\mathrm{m}, 2}$ are the maximum relaxation times of the component polymers.

We discuss the effects of $\tau_{\mathrm{m}}$ of the component polymers on the shape of the relaxation spectrum of the blend by assuming $\tau_{\mathrm{m}, 1}$ and $\tau_{\mathrm{m}, 2}$ as will

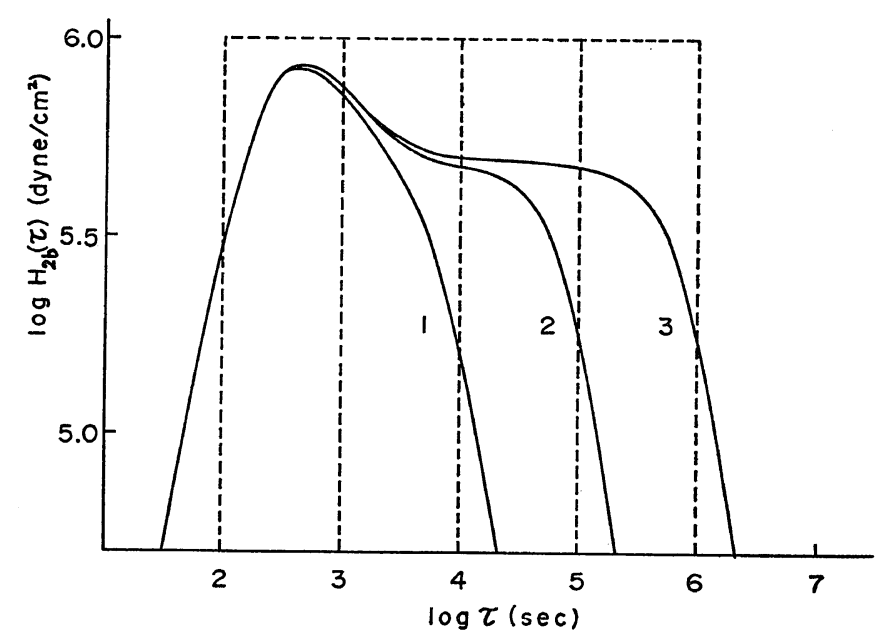

Figure 6. Relaxation spectra obtained from the linear mixing of two ideal box distributions. Dotted lines show the initial box spectra. The number in the figure corresponds to that of Table III. 
as shown in Table III.

In order to avoid complications, the values of $C_{1}, C_{2}, \tau_{\mathrm{e}}$, and $E_{\mathrm{o}}$ were assumed as $0.5,0.5$, $10^{2} \mathrm{sec}$ and $10^{6} \mathrm{dyn} / \mathrm{cm}^{2}$, respectively. The relaxation spectrum of each blend was obtained from the relaxation modulus $E_{r, \text { b1 }}(t)$ using the second approximation method (Figure 6).

It can be seen from Figure 6 that as the difference $\tau_{\mathrm{m}, 2}-\tau_{\mathrm{m}, 1}$ increases, the step-like shape in the rubbery region of the spectra becomes remarkable. This is only a simplified speculation, but satisfactorily explains the shape of the relaxation spectra of the B-2 and B-3 polyblends.

Now, for the discrete distribution of relaxation times, the relaxation modulus is written as, ${ }^{12}$

$$
E_{\mathrm{r}}(t)=E_{\mathrm{a}} \mathrm{e}^{-t / \tau_{\mathrm{a}}}+\cdots+E_{\mathrm{i}} \mathrm{e}^{-t / \tau_{\mathrm{i}}}+\cdots+E_{\mathrm{m}} \mathrm{e}^{-t / \tau_{\mathrm{m}}}
$$

where $\tau_{\mathrm{a}}<\cdots<\tau_{\mathrm{i}}<\cdots<\tau_{\mathrm{m}}$. Thus, the steadyflow viscosity is given by,

$$
\begin{aligned}
\eta_{t}= & \int E_{\mathrm{r}}(t) \mathrm{d} t \\
= & \int_{0}^{\infty} E_{\mathrm{a}} \mathrm{e}^{-t / \tau_{\mathrm{a}}} \mathrm{d} t+\cdots+\int_{0}^{\infty} E_{\mathrm{i}} \mathrm{e}^{-t / \tau_{\mathrm{i}}} \mathrm{d} t+\cdots \\
& +\int_{0}^{\infty} E_{\mathrm{m}-1} \mathrm{e}^{-t / \tau_{\mathrm{m}-1}} \mathrm{~d} t+\cdots \\
& +\int_{0}^{\infty} E_{\mathrm{m}} \mathrm{e}^{-t / \tau_{\mathrm{m}}} \mathrm{d} t \\
= & E_{\mathrm{a}} \tau_{\mathrm{a}}+\cdots+E_{\mathrm{i}} \tau_{\mathrm{i}}+\cdots+E_{\mathrm{m}-1} \tau_{\mathrm{m}-1}+E_{\mathrm{m}} \tau_{\mathrm{m}}
\end{aligned}
$$

Steady-state compliance $J_{\mathrm{e}}$ may be shown as follows.

$$
J_{\mathrm{e}}=\frac{1}{\eta_{\mathrm{t}}{ }^{2}} \int_{0}^{\infty} \int_{t^{*}}^{\infty} E_{\mathrm{r}}(t) \mathrm{d} t \mathrm{~d} t^{*}
$$

Combining eq 9 and 11 ,

$$
\begin{aligned}
J_{\mathrm{e}}= & \frac{1}{\eta_{\mathrm{t}}{ }^{2}}\left(E_{\mathrm{a}} \tau_{\mathrm{a}}{ }^{2}+\cdots+E_{\mathrm{i}} \tau_{\mathrm{i}}{ }^{2}+\cdots\right. \\
& \left.+E_{\mathrm{m}-1} \tau_{\mathrm{m}-1}+E_{\mathrm{m}} \tau_{\mathrm{m}}{ }^{2}\right)
\end{aligned}
$$

The molecular weights, the values of $\bar{M}_{w} / \bar{M}_{n}$, the maximum relaxation times $\tau_{\mathrm{m}}$ and their distributions $E_{\mathrm{m}}$ which were calculated from the superposed master curves using the well-known procedure $X,{ }^{12}$ steady-flow viscosities $\eta_{\mathrm{t}}$, and steady-state compliances $J_{\mathrm{e}}$ are summarized in Table IV. $\eta_{t}$ and $J_{\mathrm{e}}$ were obtained from master curves by means of eq 9 and 11 .

With the use of the data shown in Table IV, the following figures were plotted. First, the the relation between the maximum relaxation time and the weight-average molecular weight $\bar{M}_{w}$ is shown in Figure 7 . The straight line approximately fits the points in Figure 7 for the B-1, B-2, and B-3 blends. Compared with the case of monodisperse PSt, however, some deviation from the line was observed in these systems. of polyblends. The slope of this straight line is about 3.9 which fairly agrees with the ideal value $3.4 .^{5}$

Steady-flow viscosities of the same samples were also plotted against the weight-average molecular weights $\bar{M}_{w}$. The deviation from the straight line was also observed more clearly for the polyblends than for the monodisperse PSt. The slope of this line is about 4.1 which is a little greater than the ideal value 3.4 .

In Figures 9 and 10, we plotted the relation between $\eta_{\mathrm{t}}$ vs. $E_{\mathrm{m}} \tau_{\mathrm{m}}$ and $\int_{0}^{\infty} \int_{t^{*}}^{\infty} E_{\mathrm{r}}(t) \mathrm{d} t \mathrm{~d} t^{*} \quad v s$.

Table IV. Viscoelastic parameters for monodisperse and blended polystyrenes

\begin{tabular}{lcccc}
\hline Sample & $E_{\mathrm{m}} \times 10^{-5}, \mathrm{dyn} / \mathrm{cm}^{2}$ & $\tau_{\mathrm{m}}, \mathrm{sec}$ & $\eta_{\mathrm{t}}$, poise & $J_{\mathrm{e}} \times 10^{6}, \mathrm{~cm}^{2} / \mathrm{dyn}$ \\
\hline MD-2 & 6.41 & $4.07 \times 10^{2}$ & $8.68 \times 10^{8}$ & 0.53 \\
MD-3 & 6.46 & $1.97 \times 10^{4}$ & $4.12 \times 10^{10}$ & 0.53 \\
MD-4 & 7.95 & $1.18 \times 10^{6}$ & $2.64 \times 10^{11}$ & 0.35 \\
B-1-1 & 5.89 & $1.27 \times 10^{5}$ & $1.86 \times 10^{12}$ & 0.49 \\
B-1-2 & 3.64 & $7.15 \times 10^{3}$ & $5.56 \times 10^{9}$ & 0.97 \\
B-1-3 & 1.62 & $2.57 \times 10^{3}$ & $1.56 \times 10^{9}$ & 2.91 \\
B-2-1 & 4.08 & $1.73 \times 10^{5}$ & $1.91 \times 10^{11}$ & 0.71 \\
B-2-2 & 3.39 & $9.33 \times 10^{4}$ & $8.62 \times 10^{10}$ & 0.92 \\
B-2-3 & 6.76 & $4.34 \times 10^{4}$ & $1.34 \times 10^{10}$ & 3.18 \\
B-3-2 & 2.69 & $3.81 \times 10^{5}$ & $1.74 \times 10^{11}$ & 1.18 \\
B-3-3 & 14.6 & $1.25 \times 10^{5}$ & $1.62 \times 10^{11}$ & 0.50 \\
\hline
\end{tabular}


K. Murakami, K. Ono, K. Shimna, T. Ueno, and M. Matsuo

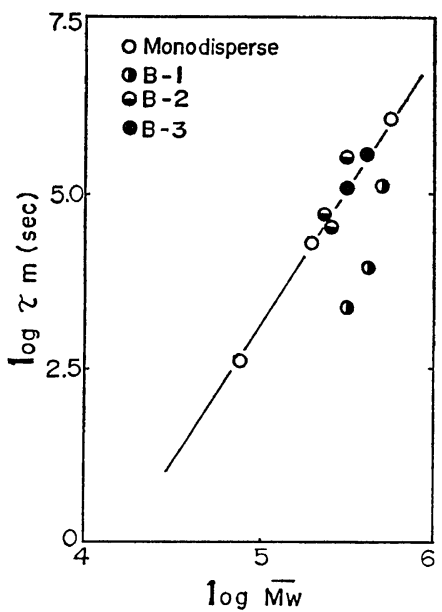

Figure 7. Molecular weight dependence of the maximum relaxation time $\tau_{\mathrm{m}}$.

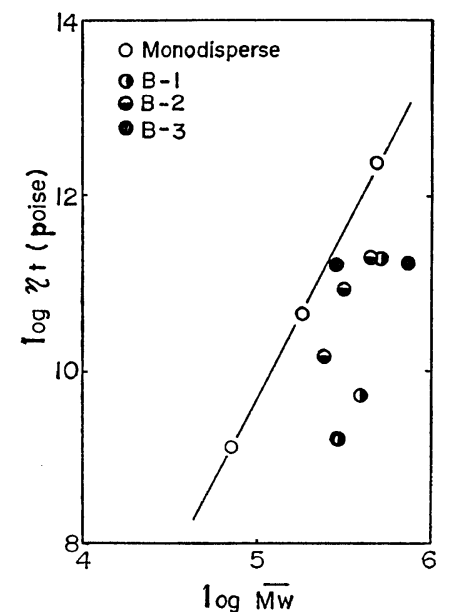

Figure 8. Molecular weight dependence of the steady-flow viscosity $\eta_{\mathrm{t}}$.

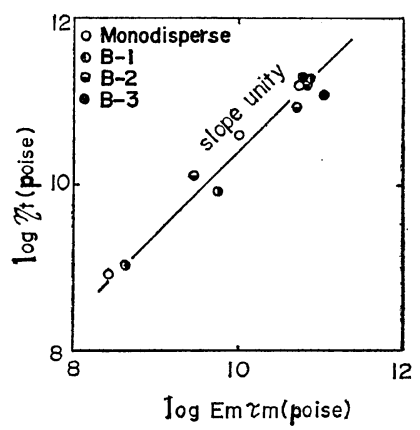

Figure 9. Relation between the steady-flow viscosity $\eta_{\mathrm{t}}$ and $E_{\mathrm{m}} \tau_{\mathrm{m}}$.

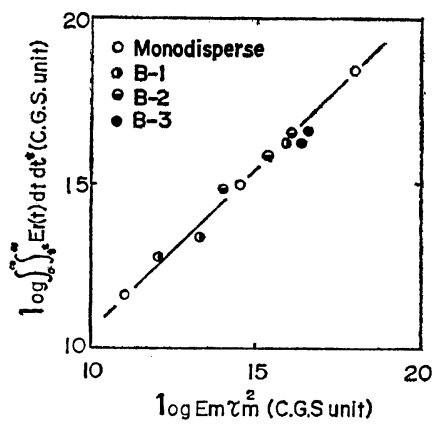

Figure 10. Relation between $\int_{0}^{\infty} \int_{t^{*}}^{\infty} E_{1}(t) \mathrm{d} t \mathrm{~d} t^{*}$ and $E_{\mathrm{m}} \tau_{\mathrm{m}}^{2}$.

$E_{\mathrm{m}} \tau_{\mathrm{m}}{ }^{2}$ using the data shown in Table IV. Obviously, any differences between the monodisperse polymers and the polyblends were not observed in these figures and the straight lines with slope 1 fitted in any case. From Figure 10 , it may be said that eq 12 is reduced to the following approximate expression.

$$
J_{\mathrm{e}}=\frac{1}{\eta_{\mathrm{t}}{ }^{2}} E_{\mathrm{m}} \tau_{\mathrm{m}}{ }^{2}
$$

Now, for an ideal box distribution, eq 10 is reduced to

$$
\eta_{\mathrm{t}}=E_{\mathrm{m}} \tau_{\mathrm{m}}
$$

Substituting eq 14 into eq 13 , we obtain

$$
J_{\mathrm{e}}=\frac{1}{E_{\mathrm{m}}}
$$

As shown in Figure 9, it may be more practical to relate $\eta_{\mathrm{t}}$ with $E_{\mathrm{m}} \tau_{\mathrm{m}}$ using a parameter $\alpha$

$$
\alpha=E_{\mathrm{m}} \tau_{\mathrm{m}} / \eta_{\mathrm{t}}
$$

It was suggested previously ${ }^{8}$ that the parameter $\alpha$ has some relation to the molecular weight distribution, but such a relation was not observed in this work. Substituting eq 16 into eq 13 , we obtain

$$
J_{\mathrm{e}}=\alpha^{2} / E_{\mathrm{m}}
$$

The relations between the molecular weights of the samples shown in Table IV and the viscoelastic parameters $\tau_{\mathrm{m}}, E_{\mathrm{m}}, \eta_{\mathrm{t}}$, and $J_{\mathrm{e}}$ are summarized in Table $\mathrm{V}$ and compared with the experimental data of other workers. 
Stress Relaxation of Blended Polystyrenes

Table V. Molecular weight dependences of viscoelastic parameters obtained by theories and experiments

\begin{tabular}{llllll}
\hline & $E_{\mathrm{m}}$ & $\eta_{\mathrm{t}}$ & $\tau_{\mathrm{m}}$ & $\tau_{\mathrm{m}} / \eta_{\mathrm{t}}$ & $J_{\mathrm{e}}$ \\
\hline Rouse-Bueche theory ${ }^{1,16}$ & $M^{-1}$ & $M^{1}$ & $M^{2}$ & $M^{1}$ & $M^{1}$ \\
Modification of Ferry, Landel, and Williams & $M^{-1}$ & $M^{3.4}$ & $M^{4.4}$ & $M^{1}$ & $M^{1}$ \\
Observation (Sobue and Murakami,8) & $M^{0}$ & $M^{3.4}$ & $M^{3.4}$ & $M^{0}$ & $M^{0}$ \\
Observation (Akovali15) & $M^{0}$ & $M^{3.75}$ & $M^{3.75}$ & & $M^{0}$ \\
Observation (Tobolsky and Andrewsis) & & $M^{3.3}$ & $M^{3.3}$ & $M^{0}$ & \\
Observation of this work & $M^{0}$ & $M^{4.1}$ & $M^{3.9}$ & $M^{0}$ & $M^{0}$ \\
\hline
\end{tabular}

An important problem is whether the value $J_{\mathrm{e}}$ depends upon molecular weight or not. Judging from the present results, $J_{e}$ is independent of molecular weight and it has a rather close relation to the molecular weight distribution.

In conclusion, for monodisperse polymers clear relations between the viscoelastic parameters $\tau_{\mathrm{m}}, \eta_{\mathrm{t}}$, and the molecular weight were observed, while for polyblends these relations were not so clear. On the other hand, it was observed that the viscoelastic parameters were closely related together by eq 13 and 16 in both cases of monodisperse and blended polymers.

It must be emphasized that the investigations of the basic relations between the molecular weights of components in the mixture and the maximum relaxation times are necessary for the viscoelastic study of polymer blends. In the following treatment we shall discuss these problems.

Ninomiya ${ }^{13}$ has discussed the behavior or the relaxation modulus of the blended polymer, taking up poly(vinyl acetate) as an example and found the following linear additivity.

$$
E_{\mathrm{r}, \mathrm{b}_{1}}(t)=w_{1} E_{\mathrm{r}, 1}\left(t / \lambda_{1}\right)+w_{2} E_{\mathrm{r}, 2}\left(t / \lambda_{2}\right)
$$

where $E_{\mathrm{r}, 1}(t), E_{\mathrm{r}, 2}(t)$ and $E_{\mathrm{r}, \mathrm{b} 1}(t)$ are the relaxation moduli of polymer components, and the polymer blends, respectively, $w_{1}$ and $w_{2}$ denote the weight fraction of polymer components in the mixture, and where $\lambda_{i}$ is the shift factor of the relaxation modulus of each component. If the steady-flow viscosity is proportional to the 3.4 power of the molecular weight, the following expression will be obtained as was shown by Ninomiya. ${ }^{13}$

$$
\lambda_{\mathrm{i}}=\bar{M}_{n, \mathrm{~b} 2} / \bar{M}_{n, \mathrm{i}}
$$

Here the subscript $\mathrm{i}$ shows the polymer component 1 or 2 . $\bar{M}_{n, \mathrm{~b} 1}$ and $\bar{M}_{n, \mathrm{i}}$ denote the number-average molecular weights of the blend and a component.

On the other hand, Ferry has discussed the additivity of the relaxation spectrum using the theory of flexible chains and obtained. ${ }^{14}$

$$
\begin{aligned}
H_{\mathrm{b} 1}(\tau)= & w_{1} H_{1}\left(\tau \eta_{1} \bar{M}_{w, 1} / \eta_{\mathrm{b} 1} \bar{M}_{w, 1}\right) \\
& +w_{2} H_{2}\left(\tau \eta_{2} \bar{M}_{w, \mathrm{~b} 1} / \eta_{\mathrm{b} 1} \bar{M}_{w, 2}\right)
\end{aligned}
$$

From the relation between $\eta$ and $\bar{M}_{w}$ the following equation will be obtained.

$$
\lambda_{\mathrm{i}}=\left(\bar{M}_{w, \mathrm{~b} 1} / \bar{M}_{w, \mathrm{i}}\right)^{2.4}
$$

The value of $\lambda_{2}$ which corresponds to the horizontal shift factor of larger molecular species must be considered. If the $E_{\mathrm{r}}(t)$ curve is shifted downward as much as $\log w$ to make the $w_{2} E_{\mathrm{r}, 2}(t)$ curve coincide, the shift factor $\lambda_{2}$ may be determined. This procedure is the same as the one in which the $\log E_{\mathrm{r}, 2}(t)$ curve is horizontally shifted as much as $\log \tau_{\mathrm{m}, \mathrm{b} 1}-\log \tau_{\mathrm{m}, \mathrm{i}}$ on the $\log E_{\mathrm{r}, 2}(t)-t$ curve maintaining the value of $E_{\mathrm{r}, 2}(t)$. Thus eq 22 will be obtained.

$$
\lambda_{\mathrm{i}}=\tau_{\mathrm{m}, \mathrm{b} 1} / \tau_{\mathrm{m}, \mathrm{i}}
$$

Table VI. Comparison of the values of $\tau_{\mathrm{m}, \mathrm{b} 1} / \tau_{\mathrm{m}, 2}$, and $\lambda_{2}$

\begin{tabular}{lcc}
\hline Sample & $\log \lambda_{2}$ & $\log \left(\tau_{\left.\mathrm{m}, \mathrm{b} 1 / \tau_{\mathrm{m}, 2}\right)}\right.$ \\
\hline B-2-1 & -0.81 & -0.83 \\
B-2-2 & -1.06 & -1.10 \\
B-2-3 & -1.50 & -1.46 \\
B-3-2 & -0.89 & -0.88 \\
\hline
\end{tabular}

where $\tau_{\mathrm{m}, \mathrm{b} 1}$ is, of course, the maximum relaxation time of the polymer blend. Eq 22 is well satisfied by the experimental data as shown in Table VI.

According to eq 19, 21, and 22, the following two eq 23 and 24 may be proposed. Further- 


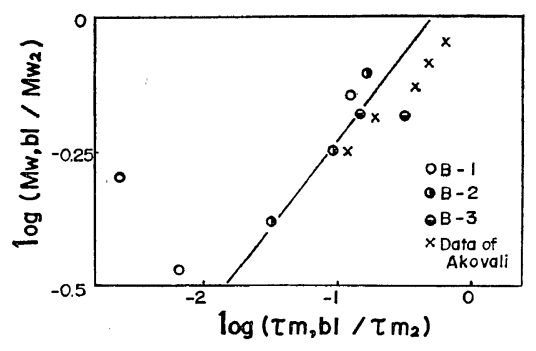

Figure 11. Relation between $\log \left(\tau_{\mathrm{m}, 1} / \tau_{\mathrm{m}, 2}\right)$ and $\log \left(\bar{M}_{w, \mathrm{~b} 1} / \bar{M}_{w, 2}\right)$.

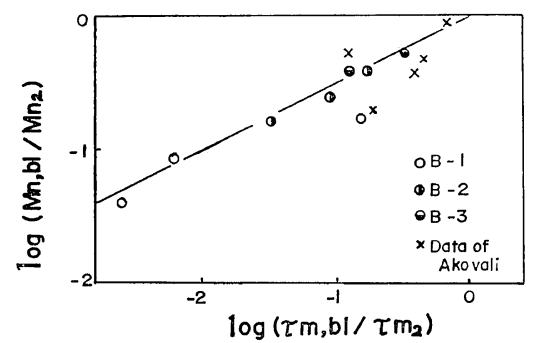

Figure 12. Relation between $\log \left(\tau_{\mathrm{m}, \mathrm{b} 1} / \tau_{\mathrm{m}, 2}\right)$ and $\log \left(\bar{M}_{n, 1} / \bar{M}_{n, 2}\right)$.

more, in terms of the results of Tobolsky and Murakami, we propose eq $25 .^{5}$

$$
\tau_{\mathrm{m}, \mathrm{b} 1} / \tau_{\mathrm{m}, \mathrm{i}}=\bar{M}_{n, \mathrm{~b} 1} / \bar{M}_{n, \mathrm{i}}
$$

For convenience, eq 23 will be called Ninomiya's equation.

$$
\tau_{\mathrm{m}, \mathrm{bi}} / \tau_{\mathrm{m}, \mathrm{i}}=\left(\bar{M}_{w, \mathrm{~b} 1} / \bar{M}_{w, \mathrm{i}}\right)^{2.4}
$$

Eq 24 will be called Ferry's equation.

$$
\tau_{\mathrm{m}, \mathrm{b} 1} / \tau_{\mathrm{m}, \mathrm{i}}=\left(\bar{M}_{w, \mathrm{b1}} / \bar{M}_{w, \mathrm{i}}\right)^{3.4}
$$

Eq 25 will be called Murakami's equation.

In order to ascertain the reliability of these three equations using the data shown in Table IV, we plotted the ratio of the maximum relaxation time of the polymer blend to that of the component polymer $\tau_{\mathrm{m}, \mathrm{b} 1} / \tau_{\mathrm{m}, \mathrm{i}}$ against the ratio of the molecular weight of the polyblend to that of the polymer component $\bar{M}_{\mathrm{b} 1} / \bar{M}_{\mathrm{i}}$ (Figures 11 and 12).

The results for the B-1, B-2, and B-3 systems of polymer blends shown in Table IV and the recent data of Akovali $^{15}$ were adopted in the $\log \bar{M}_{n, \mathrm{~b} 1} / \bar{M}_{n, 2}$ vs. $\log \left(\tau_{\mathrm{m}, \mathrm{b} 1} / \tau_{\mathrm{m}, 2}\right)$ plot. The data in Figure 11 rather scatter. The slope of the straight line which was drawn for convenience is about 3. Accordingly, it is difficult to decide which equation (eq 24 or eq 25 ) is the more correct.

Figure 12 is the plot of $\left.\log \bar{M}_{n, \mathrm{~b} 1} / \bar{M}_{n, \mathrm{i}}\right)$ against $\log \left(\tau_{\mathrm{m}, \mathrm{b} 1} / \tau_{\mathrm{m}, 2}\right)$ and the slope is about $1 / 2$. In this case, the scatter of the data is rather small compared with Figure 11 and the value of the slope is reliable. Therefore, it is difficult to apply the equation suggested by Ninomiya to this case.

As was pointed out in the case of Figures 11 and 12 , it will be an interesting problem hereafter to investigate the fundamental reasons why theoretical equations cannot be applied to the relation between the maximum relaxation time and the molecular weight in the case of blended polymers. Since all the polymers investigated in this work are the polyblends with the same kind of polymer components, the difficulty in

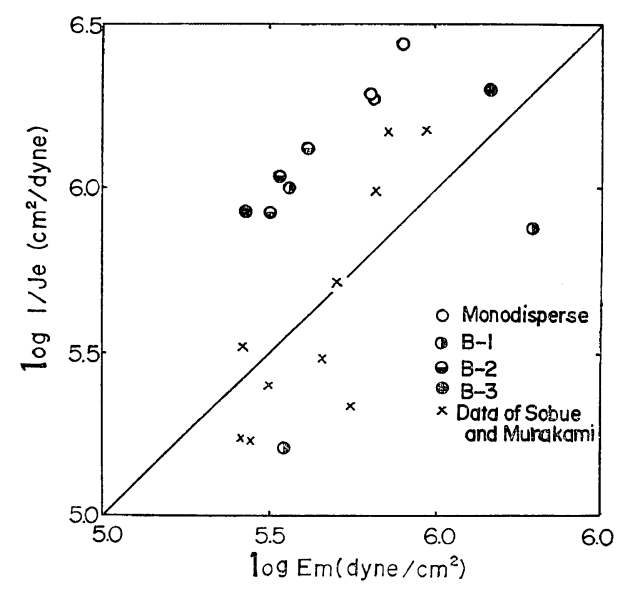

Figure 13. Plot of $1 / J_{\mathrm{e}}$ vs. $E_{\mathrm{m}}$.

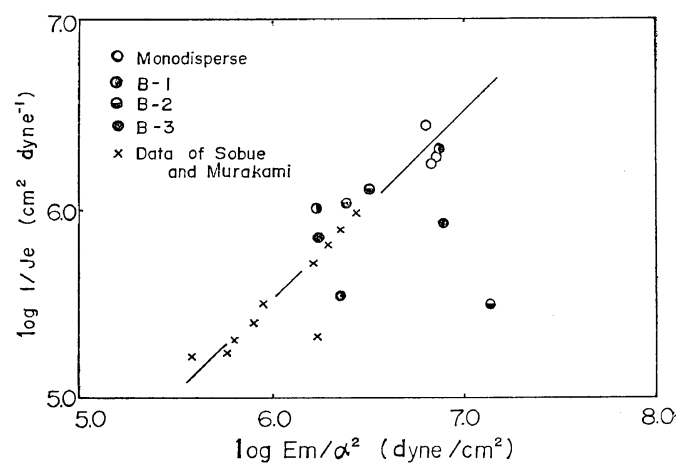

Figure 14. Plot of $1 / J_{\mathrm{e}}$ vs. $E_{\mathrm{m}} / \alpha^{2}$.

Polymer J., Vol. 2, No. 6, 1971 


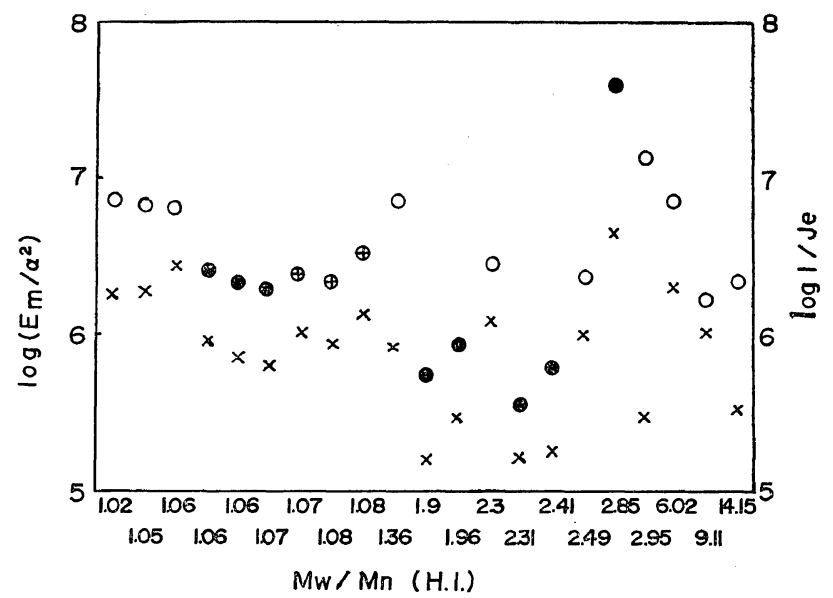

Figure 15. Relation between $E_{\mathrm{m}} / \alpha^{2}$ or $1 / J_{\mathrm{e}}$ and $\bar{M}_{w} / \bar{M}_{n}$ : $\log \left(E_{\text {In }} / \alpha^{2}\right)$ data of Sobue and Murakami ${ }^{8} ; \oplus$, data of Akovali15; $\bigcirc$, data of this work; $\times, \log \left(1 / J_{\mathrm{e}}\right)$.

blending owing to the difference in the structure or the effect of the higher structures cannot be considered. It was found that the reproducibility of the viscoelastic data of the blended polymers is very good.

Using the data in Table IV, the relation between steady-state compliance $J_{\mathrm{e}}$ and the distribution of the maximum relaxation time $E_{\mathrm{m}}$ was plotted for monodisperse and blended polymers (Figure 13).

The straight line drawn in the center of this figure denotes the approximate relation represented by eq 15 . Evidently, there is a great amount of scatter in both cases of monodisperse and blended polymers. We tried to correct this relation using the parameter $\alpha$ given by eq 16 and plotted $1 / J_{\mathrm{e}}$ against $E_{\mathrm{m}} / \alpha^{2}$ based on eq 17. The results are shown in Figure 14.

Compared with Figure 13, the straight line with the slope unity fairly fits the points in Figure 14. According to this figure, the following eq 26 may be proposed.

$$
J_{\mathrm{e}}=k\left(\alpha^{2} / E_{\mathrm{m}}\right)
$$

where $k$ is the proportional constant.

Since the values $J_{\mathrm{e}}$ and $\alpha^{2} / E_{\mathrm{m}}$ may be independent of the molecular weight as shown above, we discuss the dependence on the molecular weight distribution, as shown in Figure 15 in which the data of our previous studies, ${ }^{8}$ of Akovali $^{15}$ and the data of this work were used.
The horizontal axis shows the value of $\bar{M}_{w} / \bar{M}_{n}$, i.e., heterogeneity index (H. I.) of the PSt samples. As the vertical axis the reciprocal of the steady-state compliance $1 / J_{\theta}$ or the corresponding $E_{\mathrm{m}} / \alpha^{2}$ was taken.

In a previous report, ${ }^{8}$ it was qualitatively found that as the value $\bar{M}_{w} / \bar{M}_{n}$ increases, $1 / J_{\mathrm{e}}$ and $E_{\mathrm{m}}$ decrease. In this study, however, $J_{\mathrm{e}}$ has not any clear relation with molecular weight distribution as is shown in Figure 15.

\section{REFERENCES}

1. P. E. Rouse, J. Chem. Phys., 21, 1272 (1953).

2. B. H. Zimm, ibid., 24, 269 (1956).

3. K. Ninomiya and H. Fujita, J. Colloid Sci., 12, 204 (1957).

4. H. Sobue and K. Murakami, J. Polym. Sci., Part A-1, 1, 2039 (1963).

5. A. V. Tobolsky and K. Murakami, J. Colloid Sci., 15, 282 (1960).

6. A. V. Tobolsky, J. Amer. Chem. Soc., 74, 3786 (1952); J. Appl. Phys., 27, 673 (1956).

7. A. V. Tobolsky and K. Murakami, J. Polym. Sci., 47, 55 (1960).

8. H. Sobue and K. Murakami, ibid., 51, s29 (1961).

9. B. Zimm, P. Outer, and C. Corr, J. Chem. Phys., 18, 830 (1950).

10. T. G Fox and P. J. Flory, J. Polym. Sci., 14, 319 (1954).

11. J. D. Ferry and M. L. Williams, J. Colloid Sci., 7, 347 (1952). 
K. Murakami, K. Ono, K. Shima, T. Ueno, and M. Matsuo

12. A. V. Tobolsky and K. Murakami, J. Polym. 15. G. Akovali, J. Polym. Sci., Part A, 2, 5 (1967). Sci., 40, 443 (1959).

16. F. Bueche, J. Chem. Phys., 22, 603 (1954).

13. K. Ninomiya, J. Colloid Sci., 14, 49 (1959).

14. J. D. Ferry, "Viscoelastic Properties of Polymers", John Wiley \& Sons, Inc., New York, N. Y., 1961, p 170.

17. J. D. Ferry, R. F. Landel, and M. L. Williams, J. Appl. Phys., 26, 359 (1955).

18. R. D. Andrews and A. V. Tobolsky, J. Polym. Sci., 7, 221 (1951). 